\title{
Supporting information for Interplay of Nanoparticle Rigidity and its Translocation Ability through Cell Membrane
}

\author{
Liuyang Zhang ${ }^{1 *}$, Hongmin $\mathrm{Chen}^{2}$, Jin $\mathrm{Xie}^{3}$, Matthew Becton ${ }^{4}$ and Xianqiao Wang ${ }^{4 *}$ \\ ${ }^{1}$ State Key Laboratory for Manufacturing Systems Engineering, Xi'an Jiaotong University, Xi'an, Shaanxi, 710049, \\ China \\ ${ }^{2}$ State Key Laboratory of Molecular Vaccinology and Molecular Diagnostics \& Center for Molecular Imaging and \\ Translational Medicine, School of Public Health, Xiamen University, Xiamen, 361102, China \\ ${ }^{3}$ Department of Chemistry and Bioimaging Research Center, University of Georgia, Athens, GA 30602, USA \\ ${ }^{4}$ College of Engineering, University of Georgia, Athens, GA 30602, USA \\ *Corresponding author: liuyangzhang@xjtu.edu.cn, Tel:(+86)2983395028,
} xqwang@uga.edu, Tel: (+1)7065426251

\section{Experimental Section}

\section{Materials}

Citrated gold nanoparticles (Lot No.: MKCB4936 (50 nm), MKCB2015V (20 nm)) and dopamine hydrochloride (Lot No.: BCBM2585V), Trizma base (Lot No.: 110M54211) and FITC (Lot No.: BCBF2730V) were purchased from Sigma-Aldrich. Ultrapure water $(18.2 \mathrm{M} \Omega \cdot \mathrm{cm})$ was used in all experiments.

\section{Synthesis of polydopamine coated gold nanoparticles (Au@PDA)}

$13 \mu \mathrm{L}$ of citrated gold nanoparticles (Au NPs, size $20 \mathrm{~nm}$ and $50 \mathrm{~nm}$, Sigma Aldrich) were added into $500 \mu \mathrm{L}$ of Tris buffer $(10 \mathrm{mM}$ at $\mathrm{pH}=8.5)$ and sonicated for $10 \mathrm{~min}$. Then, $500 \mu \mathrm{L}$ of dopamine solution were added. The solution was shaken at room temperature at 900 RPM for 1 h. For $20 \mathrm{~nm} \mathrm{Au} \mathrm{NPs,} 0.40 \mathrm{mg} / \mathrm{mL}$ dopamine was used, and for $50 \mathrm{~nm} \mathrm{Au} \mathrm{NPs,} 0.10 \mathrm{mg} / \mathrm{ml}$ dopamine was used. After $1 \mathrm{~h}$, the nanoparticles were spun down at 14,000 RPM for 20 min and the pellet was resuspended in ultrapure $\mathrm{H}_{2} \mathrm{O}$; repeated 3 times.

\section{FITC-conjugated Au@PDA}

For FITC conjugation, $10 \mu \mathrm{L}$ of FITC solution in dimethyl sulfoxide $(1 \mathrm{mg} / \mathrm{mL})$ were mixed with 1mL of Au@PDA (10 10 particles/mL) and shaken at 1000 RPM. After 30 min, the solution was centrifuged at 14,000 RPM for 20 min and the pellet was resuspended in ultrapure $\mathrm{H}_{2} \mathrm{O}$; repeated 3 times. 


\section{Characterization of Au@PDA}

TEM was carried out on an FEI Tecnai20 transmission electron microscope operating at an accelerating voltage of $200 \mathrm{kV}$. Particle size distribution and surface charge measurements were carried out on a Malvern Zetasizer Nano ZS system. Fluorescent measurement was carried out on a Hitachi F-7000 spectrophotometer.

\section{Cell uptake}

4T1, murine mammary carcinoma $\left(10^{6}\right.$ cells) were seeded in Lab-Tek Chamber slides (Thermo Fisher) in RPMI 1640 medium at $37^{\circ} \mathrm{C}$ and 5\% $\mathrm{CO}_{2}$. After $24 \mathrm{~h}$ incubation, FITC-conjugated Au@PDA NPs ( $10^{8} \mathrm{Au}$ NPs) were added and incubated. After $4 \mathrm{~h}$ incubation, the slides were washed into wells with PBS three times to remove the free FITC-conjugated Au@PDA NPs. Then, fluorescent imaging was obtained using an Olympus IX71 fluorescent microscope. 4T1 cells with no added NPs acted as a control. ImageJ was employed for the analysis of fluorescence intensity based on total FITC fluorescent intensities and cell numbers.
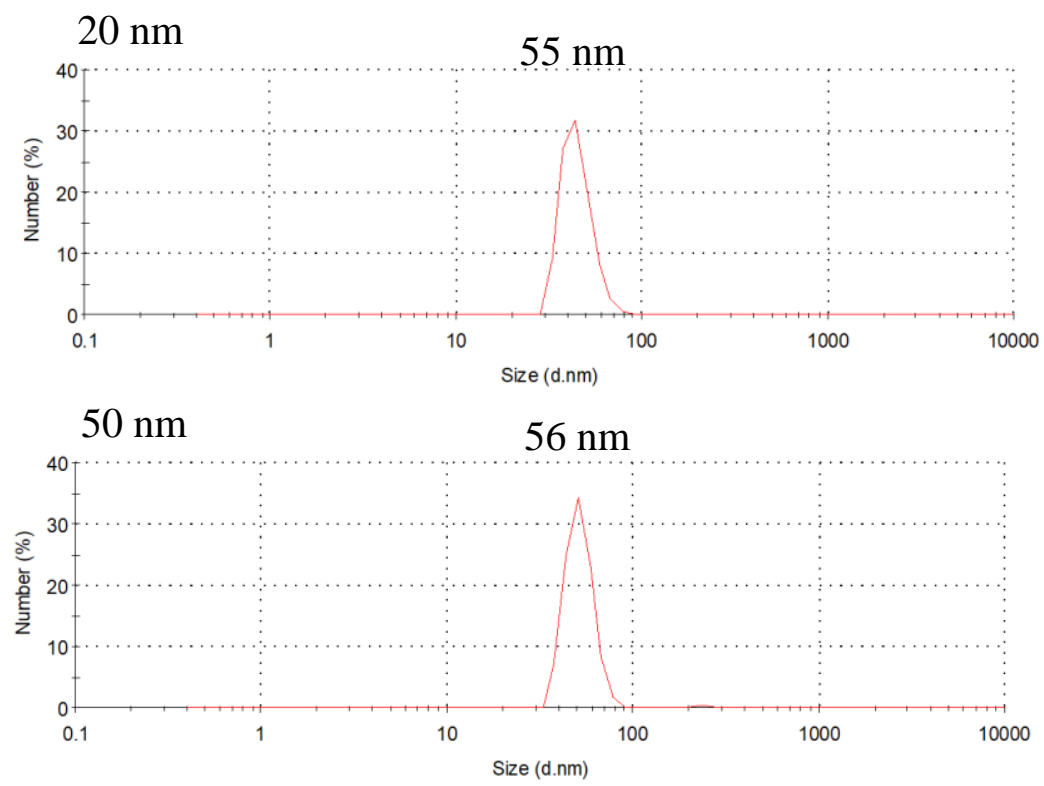

Figure S1: DLS analysis of Au@PDA nanoparticles. 

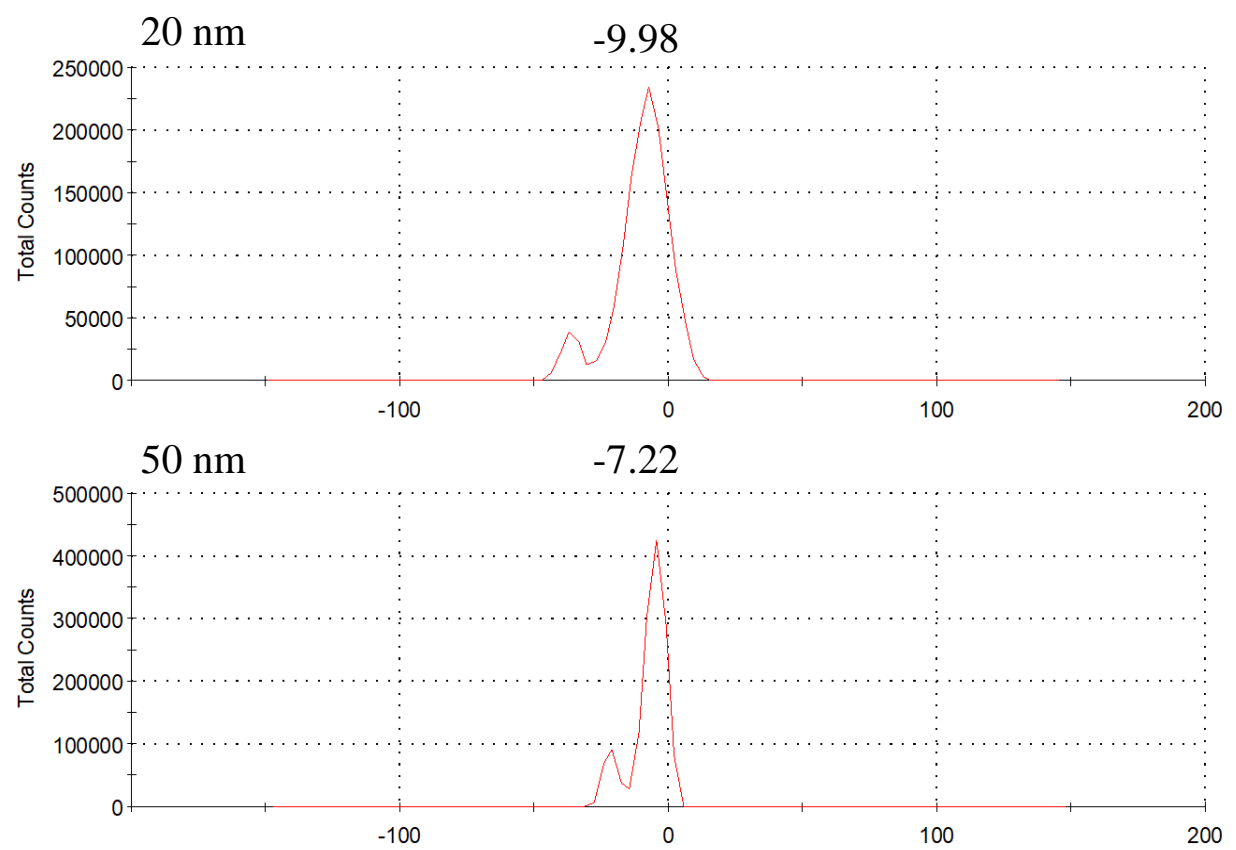

Figure S2: Surface charge analysis of Au@PDA nanoparticles.

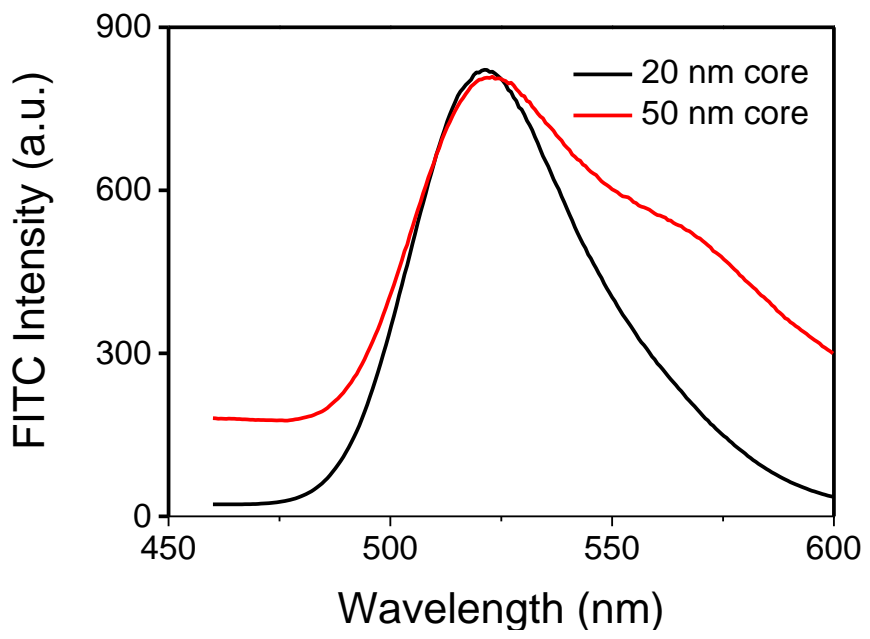

Figure S3: Before adding NPs into the cells, we measured the fluorescent intensity to confirm that the same Au nanoparticle number was used.

\section{Computational Model and Methodology}

Cooke and Deserno's solvent-free model for lipid bilayers is applied based on an attractive potential, ${ }^{1}$ which can help reduce simulation time up to $90 \%$ as compared to a model with 
explicit solvents. Each lipid molecule is represented by one head bead followed by two tail beads. The following potentials are used in the simulation to describe the interactions between beads:

The size of each lipid bead is fixed via a Weeks-Chandler-Andersen potential

$$
U_{W C A}=4 \epsilon\left[\left(\frac{b}{r_{i j}}\right)^{12}-\left(\frac{b}{r_{i j}}\right)^{6}+\frac{1}{4}\right], r_{i j}<r_{c}=\sqrt[6]{2} b
$$

with the cutoff radius $r_{c}=\sqrt[6]{2} b$, where $b$ is the finite distance, $\epsilon$ is the depth of the potential well, and $r_{i j}$ is distance between two beads $i$ and $j$. In order to ensure the cylindrical lipid shape, $b$ is set as $b_{\text {head,head }}=b_{\text {head,tail }}=0.95 \sigma$ and $b_{\text {tail,tail }}=1.0 \sigma$. Three beads in a lipid molecule are linked by two FENE bonds

$$
U_{F E N E}=\sum_{b o n d s}-\frac{1}{2} k_{b e} R_{\text {max }}^{2} \ln \left(1-\frac{r^{2}}{R_{\text {max }}^{2}}\right)
$$

with rigidity $k_{b e}=30 \varepsilon / \sigma^{2}$ and divergence length $R_{\max }=1.5 \sigma$, where $r$ is bond length. Each lipid is straightened by a harmonic spring

$$
U_{\text {stretching }}=\sum_{\text {bonds }} \boldsymbol{k}_{b s}\left(r-r_{0}\right)^{2}
$$

with bending rigidity $k_{b s}=10 \varepsilon / \sigma^{2}$ and equilibrium length $r_{0}=4 \sigma$ between the head bead and the end tail bead. The absence of explicit solvents and the hydrophobic effect is compensated by an attractive interaction between the tail beads. The potential we use to account for this hydrophobic effect is

$$
U_{c o s}=\left\{\begin{array}{cc}
-\varepsilon, & r<r_{c} \\
-\varepsilon \cos ^{2}\left[\pi\left(r-r_{c}\right) / 2 w\right], & r_{c} \leq r \leq r_{c}+w \\
0, & r>r_{c}+w
\end{array}\right.
$$

which describes an attractive potential with depth $\varepsilon$ that smoothly tapers to zero for $r>r_{c}$, where $w$ is the decay coefficient set as $1.6 \sigma$. The interaction between NPs and lipid heads in cell membranes are described by the Lennard-Jones potential function that can well describe the interaction between atoms at the micro- or nano-level.

$$
U_{L J}=4 \epsilon\left[\left(\frac{b}{r_{i j}}\right)^{12}-\left(\frac{b}{r_{i j}}\right)^{6}\right], r_{i j}<r_{c}=2.5 \sigma
$$

where $r_{i j}$ is the distance between beads $i$ and $j$. In our simulations, the unit of length is taken to be $\sigma$, the unit of mass is that of the lipid bead $m$, and the unit of energy is $\varepsilon=k_{B} T / 1.1=$ 
$2.2 \mathrm{~kJ} / \mathrm{mol}$ as previously stated in Cooke and Deserno's paper. All other quantities are expressed in terms of these basic units. These reduced basic units can be converted to SI by examining the membrane thickness and the lipid diffusion coefficient from experiments. The simulated thickness value of the bilayer is $5 \sigma$ and the effective time scale of the simulation can be determined from the simulated lateral diffusion constants of lipid bilayers. By comparison with typical experimental values, it can be shown that the basic length unit $\sigma=1 \mathrm{~nm}$ and the basic time unit $\tau=15 n s$ in physical units. The dimension of the simulation box is $100 \sigma(x) \times$ $100 \sigma(y) \times 100 \sigma(z)$. Periodic boundary conditions are applied along all directions for the simulation box. We use a velocity-Verlet algorithm to perform time integration, and a Langevin thermostat to control the system temperature. The time step for integration is $\Delta t=0.01 \tau$. During the process of NP internalization, the simulation box in the $x$-and $y$-axes can adjust to achieve a set tension on the lipid membrane, while the height of the box along the $z$-axis remains constant. Table S1 details the interaction parameters for the bilayer membrane and coated NPs. All simulations are performed based on the Sandia National Laboratory LAMMPS package. ${ }^{2}$

Table S1. Interaction parameters for the bilayer membrane and coated NPs

\begin{tabular}{l|l|c|c}
\hline \multicolumn{1}{c|}{ Bead type } & \multicolumn{1}{c|}{ Bead type } & Interaction Potential & Parameters \\
\hline Lipid head (membrane) & Lipid head (membrane) & $U_{W C A}$ & $b=0.95 \sigma$ \\
\hline Lipid head (membrane) & Lipid tail (membrane) & $U_{W C A}$ & $b=0.95 \sigma$ \\
\hline Lipid tail (membrane) & Lipid tail (membrane) & $U_{C O S}$ & $b=\sigma, w=1.6 \sigma$ \\
\hline Lipid head (vesicle) & Lipid head (vesicle) & $U_{W C A}$ & $b=0.95 \sigma$ \\
\hline Lipid head (vesicle) & Lipid tail (vesicle) & $U_{W C A}$ & $b=0.95 \sigma$ \\
\hline Lipid tail (vesicle) & Lipid tail (vesicle) & $U_{c o s}$ & $b=\sigma, w=1.6 \sigma$ \\
\hline Lipid head (membrane) & Lipid head (vesicle) & $U_{L J}$ & $b=\sigma$ \\
\hline Lipid head (membrane) & Lipid tail(vesicle) & $U_{W C A}$ & $b=0.95 \sigma$ \\
\hline Lipid tail (membrane) & Lipid head (vesicle) & $U_{W C A}$ & $b=0.95 \sigma$ \\
\hline Lipid tail (membrane) & Lipid tail (vesicle) & $U_{C O S}$ & $b=\sigma, w=1.6 \sigma$ \\
\hline Lipid head (membrane) & NPs & $U_{L J}$ & $b=\sigma$ \\
\hline Lipid tail (membrane) & NPs & $U_{W C A}$ & $b=0.95 \sigma$ \\
\hline Lipid head (vesicle) & NPs & $U_{L J}$ & $b=\sigma$ \\
\hline Lipid tail (vesicle) & NPs & $U_{W C A}$ & $b=0.95 \sigma$ \\
\hline NPs & NPs & $U_{L J}$ & $b=\sigma$ \\
\hline
\end{tabular}




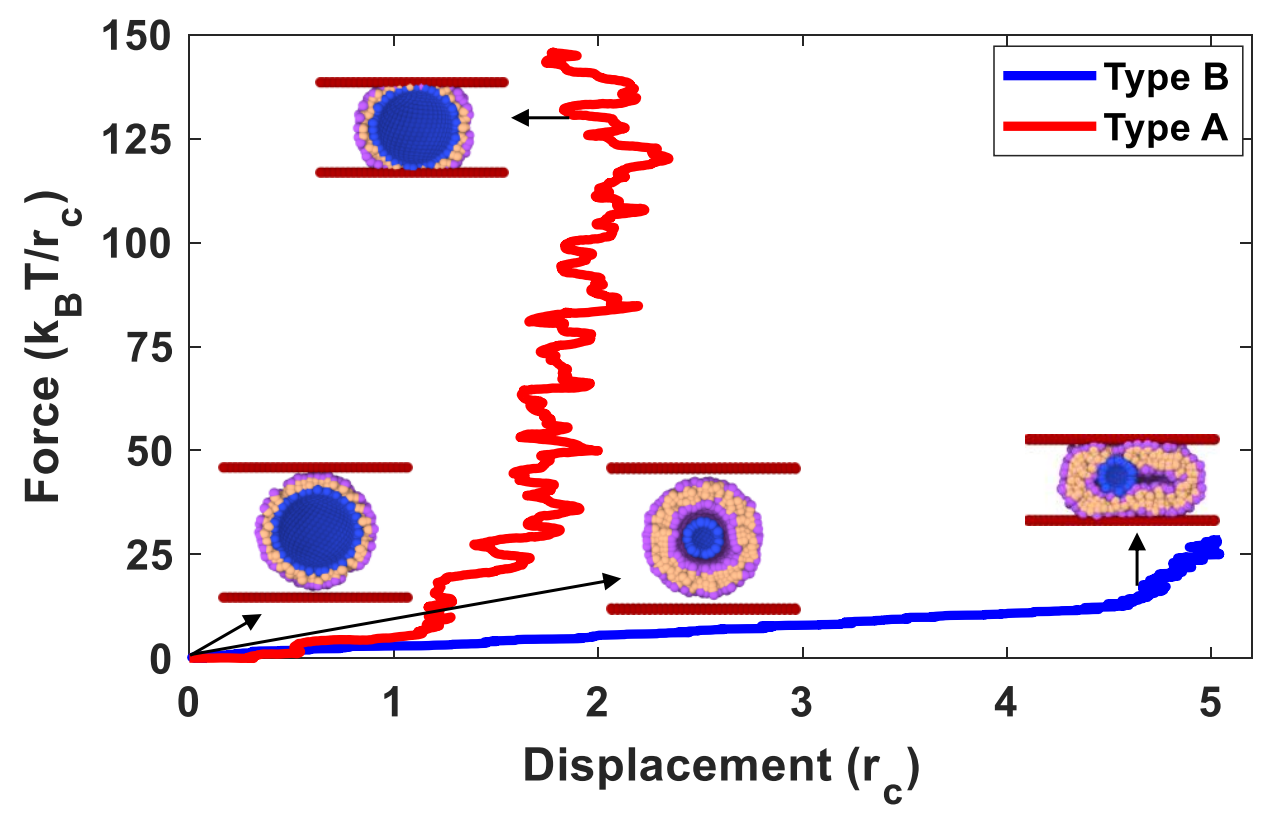

Figure S4: Force-displacement relationship of Type A and Type B NP under the compression test. As the shell thickness is around $2 \mathrm{r}_{c}$, the compressive force drastically increases when the upward indenter reaches to the core of Type A NP. For Type B NP, the compressive force slowly increases until the indenter reaches the hard core. The slope of the force-displacement curves shows the difference in the rigidity of these two types of NPs.

\section{Reference}

1. Cooke, I. R.; Deserno, M., Solvent-free model for self-assembling fluid bilayer membranes: stabilization of the fluid phase based on broad attractive tail potentials. J Chem Phys 2005, 123 (22), 224710.

2. Plimpton, S., Fast Parallel Algorithms for Short-Range Molecular Dynamics. J. Comput. Phys. 1995, $117(1), 1-19$. 